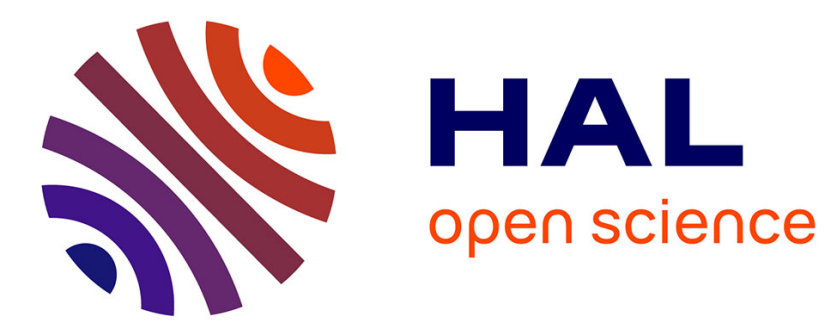

\title{
Mechanical behaviour near grain boundaries of He-implanted UO 2 ceramic polycrystals
}

Mona Ibrahim, É. Castelier, H. Palancher, Michel Bornert, Sabine Caré, J.-S. Micha

\section{- To cite this version:}

Mona Ibrahim, É. Castelier, H. Palancher, Michel Bornert, Sabine Caré, et al.. Mechanical behaviour near grain boundaries of He-implanted UO 2 ceramic polycrystals. Journal of Nuclear Materials, 2017, 483, pp.13-20. 10.1016/j.jnucmat.2016.10.044 . hal-01711377

\section{HAL Id: hal-01711377 \\ https://hal-enpc.archives-ouvertes.fr/hal-01711377}

Submitted on 24 Apr 2019

HAL is a multi-disciplinary open access archive for the deposit and dissemination of scientific research documents, whether they are published or not. The documents may come from teaching and research institutions in France or abroad, or from public or private research centers.
L'archive ouverte pluridisciplinaire HAL, est destinée au dépôt et à la diffusion de documents scientifiques de niveau recherche, publiés ou non, émanant des établissements d'enseignement et de recherche français ou étrangers, des laboratoires publics ou privés. 


\title{
Mechanical behaviour near grain boundaries of He-implanted $\mathrm{UO}_{2}$ ceramic polycrystals
}

\author{
M. Ibrahim ${ }^{\mathrm{a}, \mathrm{b}, *}$, É. Castelier ${ }^{\mathrm{a}}$, H. Palancher ${ }^{\mathrm{a}}$, M. Bornert ${ }^{\mathrm{b}}$, S. Caré ${ }^{\mathrm{b}}$, \\ J.-S. Micha ${ }^{\mathrm{c}}$ \\ ${ }^{a} C E A$, DEN, DEC, Centre de Cadarache, 13108 St Paul lez Durance, France \\ ${ }^{b}$ Laboratoire Navier, UMR 8205, École des Ponts, IFSTTAR, CNRS, UPE, \\ Champs-sur-Marne, France \\ ${ }^{c}$ CEA, INAC, 38054 Grenoble Cedex 9 , France
}

\begin{abstract}
For studying the micromechanical behaviour of $\mathrm{UO}_{2}$ and characterising the intergranular interaction, polycrystals are implanted with helium ions, inducing strains in a thin surface layer. Laue X-ray micro-diffraction is used to measure the strain field in this implanted layer with a spatial resolution of about 1 micrometer. It allows a 2D mapping of the strain field in a dozen of grains. These measurements show that the induced strain depends mainly on the crystal orientation, and can be evaluated by a semi-analytical mechanical model. A mechanical interaction of the neighbouring grains has also been evidenced near the grain boundaries, which has been well reproduced by a finite element model. This interaction is shown to increase with the implantation energy (i.e. the implantation depth): it can be neglected at low implantation energy $(60 \mathrm{keV})$, but not at higher energy $(500 \mathrm{keV})$.
\end{abstract}

Keywords: Mechanical analysis, Polycrystal, Stress singularity, Light ion implantation, X-ray micro-diffraction, 2D strain mapping

\section{Introduction}

Light-ion implantation techniques can be used for both fundamental studies and technological purposes. For instance, in the microelectronics industry,

\footnotetext{
*Corresponding author

Email address: marcelle.ibrahim@cea.fr (M. Ibrahim)
} 
these techniques are used to change the physical properties of the thin implanted layer [1]. For nuclear materials, they are used to study the effect of radiations on different materials [2], while avoiding costly neutron irritations that make the samples highly radioactive. The studied irradiation damages include point and extended crystallographic defects [3], cavities [4], etc.

This paper will focus on another use of these techniques: ion implantation induces a swelling, loading mechanically a surface layer. It is then possible to access the material behaviour by analysing the resulting strain field. The first studies of this kind dealt with single crystals [5], therefore probing very few crystal orientations. In these cases, the strain measurement was done by X-ray diffraction. An improvement has come from the X-ray micro-diffraction, enabled by synchrotron radiation: the micro-focused X-ray beam can reach a spatial resolution smaller than 1 micrometer $[6,7,8]$. It is now possible to implant ions in a polycrystal, alloys [9] or ceramics [10], and to measure accurately the resulting strain inside several grains (about 1000) with different orientations, or to map the strain field inside dozens of grains [11].

In single crystals, the relationship between the implantation induced swelling and the observed strain depends on the mechanical properties and on the crystal orientation regardless of the geometry [10, 12], the measurements are thus easy to interpret. In a polycrystal, the mechanical interactions of all the neighbouring grains make the mechanical analysis more difficult: how far will these interactions disturb the measurement? Can the grains be considered as independent of each other? This paper wants to answer these questions by characterising the strain field of the implanted layer, both by accurate strain field measurements and by mechanical modelling.

The samples of this study are $\mathrm{UO}_{2}$ polycrystals implanted with helium ions. Two implantation energies have been used, 60 and $500 \mathrm{keV}$, in order to vary the implantation depth. For both cases, the implantation depth is smaller than the X-rays penetration depth, producing double Laue diffraction spots: one coming from the implanted deformed layer and another from the non deformed substrate. A special Laue pattern analysis has been developed for this case [10], allowing an absolute strain measurement in the implanted layer. Some improvements have been added [11] for a better confidence, in order to measure accurately the slight variations of the strain field inside the grains and near the grain boundaries. The data coming from these methods are now interpreted by a mechanical analysis consisting of a semi-analytical model to describe the influence of the grain orientation and of finite element 
simulations to reproduce the strain measurements near the grain boundaries.

\section{Ion implantation and $\mu$-XRD}

Ion implantation. The two $\mathrm{UO}_{2}$ polycrystalline samples used in this study are disks of $8 \mathrm{~mm}$ diameter and $1 \mathrm{~mm}$ thickness, with a grain size of about $18 \mu \mathrm{m}$. They have been implanted with helium at a fluence of $10^{16}$ ions $/ \mathrm{cm}^{2}$ with two different energies: 60 and $500 \mathrm{keV}$. The implantation induces strains in a thin surface layer. In-depth characterisation using monochromatic X-ray techniques estimates the strained layer thickness to $0.4 \mu \mathrm{m}$ [13] and $1.3 \mu \mathrm{m}$ [14] for $60 \mathrm{keV}$ and $500 \mathrm{keV}$ respectively. These values are in agreement with the damage profiles estimated using SRIM software [15] for these conditions.

$X$-ray micro-diffraction. Strains in the implanted layer are measured using X-ray diffraction, with a beam size of about $1 \mu \mathrm{m}$, much smaller than the grain size, in order to characterise the strain field variations inside the grains, particularly at their boundaries. These measurements have been done with the synchrotron radiation of the European Synchrotron Radiation Facility (ESRF), at the BM32 beamline [6]. The incident beam was polychromatic (5-22 keV), producing Laue patterns on a CCD camera, containing between twenty and forty diffraction spots.

Figure 1 gives the principles of this measurement: with a penetration depth of approximately $5 \mu \mathrm{m}$ in $\mathrm{UO}_{2}$, greater than the implantation depth, the polychromatic incident beam is diffracted both by the implanted layer and the strain free substrate, producing double spots on the Laue pattern. The strain in the implanted layer can be deduced from the distance between

the two maxima of the diffraction spots, and is thus measured relatively to the strain free substrate. Contrary to other measurement techniques, for instance EBSD (Electron backscatter diffraction), where only the surface layer is probed, our Laue patterns contain a reference to the strain free material, thus providing absolute strain values that can be compared between grains of different orientations.

Laue pattern automatic analysis. The displacement of the diffraction spots is related to the displacement gradient tensor $\boldsymbol{\nabla} \boldsymbol{\xi}$ in the implanted layer, which symmetric and antisymmetric parts correspond to the strain and rotation tensors:

$$
\boldsymbol{\epsilon}=\frac{1}{2}\left(\nabla \boldsymbol{\xi}+\nabla \boldsymbol{\xi}^{T}\right), \quad \omega=\frac{1}{2}\left(\nabla \boldsymbol{\xi}-\nabla \boldsymbol{\xi}^{T}\right) .
$$


In this paper, we prefer to deal with the displacement gradient $\boldsymbol{\nabla} \boldsymbol{\xi}$ rather than the strain $\boldsymbol{\epsilon}$ because it is directly measured on the Laue patterns and it bears more information.

A special Laue pattern analysis has been developed [10] to measure the displacement gradient from these typical Laue patterns. First, an automatic image analysis procedure detects the two peaks of the diffraction spots, then, the displacement gradient is estimated by least squares techniques. This first analysis, applied on $\mathrm{UO}_{2}$ samples implanted with $60 \mathrm{keV}$ helium ions, has shown that the displacement gradient tensor $\boldsymbol{\nabla} \boldsymbol{\xi}$ mainly depends on the grain orientation. However the measurement precision was not accurate enough to measure the slight variations of the strain field inside the grains.

The proposed method has thus been improved [11]: new image analysis procedures, combined with statistical tools were developed to reduce the measurement error by a factor 2 and to correct detection errors. With the increase of the implantation energy, the strain in the implanted layer varies along the depth [14], changing the shape of the double Laue spot shown in Figure 1. A specific work has been done [16] to face this problem: for high energies (for example $500 \mathrm{keV}$ ), the strain measured on the Laue patterns corresponds to a mean value over the implantation layer. It is now possible to accurately measure the slight variations of the strain field inside each grain, for several implantation energies.

$2 D$ strain mapping. The two implanted samples have been probed on a regular grid with a $1.5 \times 2 \mu \mathrm{m}^{2}$ step along the sample surface, in order to map the strain field at the grain scale. Figure 2 presents the maps of the $\xi_{z, z}$ component of the displacement gradient, which corresponds to the strain $\epsilon_{z z}$ normal to the sample surface, for both implantation conditions. Each pixel of these maps is the result of a Laue pattern analysis. The pixels in dark grey, which represent a failed analysis, are mainly located at the grain boundaries, where the mechanical interaction of neighbouring grains alters the simple Laue spot shapes (see Figure 1), making the Laue patterns more difficult to interpret. More details on the pattern analysis, and maps of other gradient components $\xi_{i, j}$ have already been presented elsewhere [11].

The maps show that the displacement gradient for $60 \mathrm{keV}$ ion implantation is quite uniform inside the grains, depending mainly on their orientation. The strain field varies slightly inside the grains, particularly at their boundaries. This variation seems however greater for a deeper implantation $(500 \mathrm{keV})$. 


\section{Mechanical analysis}

All the features revealed by the strain field measurements will now be interpreted through a mechanical analysis: a semi-analytical mechanical model to explain the grain orientation dependence, and finite element simulations to model the mechanical interaction of neighbouring grains.

Mechanical model. The implantation of ions in a sample induces a stress free swelling of the implanted layer. In a cubic material as $\mathrm{UO}_{2}$, this swelling does not have a preferred orientation, and can thus be considered as isotropic:

$$
\boldsymbol{\epsilon}_{s}=\left(\begin{array}{ccc}
s / 3 & 0 & 0 \\
0 & s / 3 & 0 \\
0 & 0 & s / 3
\end{array}\right)
$$

where $s / 3$ denotes the linear free swelling, which can vary with the implantation depth $z[13,14]$.

This swelling loads mechanically the sample surface, producing stress and strain fields. In the elastic domain, the stress $\boldsymbol{\sigma}$, strain $\boldsymbol{\epsilon}$ and stress free strain $\boldsymbol{\epsilon}_{s}$ are related by Hook's Law:

$$
\boldsymbol{\sigma}=\boldsymbol{C} .\left(\boldsymbol{\epsilon}-\boldsymbol{\epsilon}_{s}\right), \quad \text { with } \quad \boldsymbol{C}=N^{T} \cdot \boldsymbol{C}_{0} \cdot N
$$

where the stiffness tensor $\boldsymbol{C}$ depends on the stiffness tensor $\boldsymbol{C}_{0}$ in the reference frame, and the crystal orientation through the orientation matrix $N$. For cubic materials, like $\mathrm{UO}_{2}$, the tensor $\boldsymbol{C}_{0}$ depends on three elastic constants: $C_{11}, C_{12}, C_{44}$ in Voigt notations.

This mechanical model must be completed by boundary conditions: the sample is at rest, no forces acting at any external surfaces. Now, we can calculate the strain of implanted samples, first using a simplified model, then using finite elements.

Semi-Analytical model. A simple solution of the mechanical Equation (1) has already been presented in details [10]: the displacement $\boldsymbol{\xi}$ varies only according to the depth $z$, which leads to the following form of the displacement gradient, with only three non zero components:

$$
\boldsymbol{\nabla} \boldsymbol{\xi}=\left(\begin{array}{ccc}
0 & 0 & \xi_{x, z} \\
0 & 0 & \xi_{y, z} \\
0 & 0 & \xi_{z, z}
\end{array}\right) .
$$


This solution does not verify rigorously the boundary conditions, because it involves forces at the circumference of the implanted layer. To counterbalance these forces, a slight bending of the sample must be added, which is actually due to the swelling of the upper implanted layer. However, this additional bending can be neglected: its relative contribution to the strain corresponds to the ratio of the implanted layer thickness $(<1.3 \mu \mathrm{m}$ in our conditions) over the sample thickness $(1 \mathrm{~mm})$, i.e. about $10^{-3}$.

Far from the circumference, the proposed approximation is thus very precise, provided the sample is made of a unique material, i.e. a single crystal. For polycrystalline materials, it can be used on the different grains as if they were independent from each other. We call this approximation the independent grains model. It has been successfully applied [10] to model the strain of about 800 grains of different orientation in a polycrystal sample implanted with $60 \mathrm{keV}$ ions, and to estimate the stress free swelling $s$ in the implanted layer. It is about $0.48 \%$ for an implantation fluence of $10^{16}$ ions $/ \mathrm{cm}^{2}$.

In the experimental maps in Figure 2, the quasi uniformity of the strain field inside the grains indicates that this approximation is adapted for both samples. However, the grains have a mechanical interaction, and the strain field is actually more complex, specially near the grain boundaries. These variations increase with the implantation energies, from 60 to $500 \mathrm{keV}$. Mechanical simulations based on finite elements will help to interpret these mechanical interactions.

Finite element modelling. In order to study the mechanical grains interaction, we focus only on two neighbouring grains. The finite element calculations are therefore done on the geometry represented in Figure 3. It is possible to vary separately several parameters: the implantation layer thickness $d h$, the boundary inclination $\alpha$, or the grains orientations. The modelling is characterised by the following features:

- The stress free swelling $s$ induced by the implantation varies with the implantation depth $z$. This point has already been examined elsewhere $[16,13]$. In our modelling, for simplification sake, we will suppose a uniform free swelling inside a thin surface layer of thickness $d h$. Its value is estimated from the strain measurements of about thousand grains of different orientations, using the independent grains model described above. 
- For a polycrystal, the boundary conditions are applied very far from the grains considered in our simulation. For reporting these far conditions, we choose periodical boundary conditions. Doing so, we know that we neglect the slight bending of the sample mentioned above, induced by the swelling of its upper layer. With the periodicity, the two grains of length $d$ alternate along the $x$ direction. They are infinitely wide along the $y$ direction.

- The upper and lower surfaces (for $z=0$ and $z=-h$ ) are stress free. The size $h$ is chosen large enough to eliminate the influence of the lower surface: 30 times greater than the layer thickness $d h$.

- The two grains have different orientations. The elastic constants of $\mathrm{UO}_{2}\left(C_{11}, C_{12}, C_{44}\right)$ are retrieved from literature [17].

Stress singularities. In this model, stress singularities appear at the intersection of the grain boundaries and the upper surface, highlighted in green on Figure 3. A stress singularity [18] is characterised by the stress field reaching infinite values near a point, according to:

$$
\sigma(r)=\frac{K}{r^{n}}
$$

where $K$ represents the stress intensity factor, $r$ the distance toward the singular point, and $n$ the singularity order. To illustrate this point, a finite element calculation has been done using the loading and boundary conditions described in the previous paragraph (Figure 3). Figure 4 shows the resulting stress profile (in red) along the $x$ direction at the sample surface. A stress singularity can be seen at each grain boundary, characterised by a discontinuity and very high values.

Singularities in this kind of configurations have been extensively studied in literature $[19,18,20]$. For elastic isotropic materials, the singularity can be studied with analytic formulae: the singularity order $n$ depends on the Young's modulus ratio of the two materials, and the angles between the free surface and the materials interface; the stress intensity factor $K$ depends on the overall geometry and loading conditions. We choose to study these singularities in our cubic material using finite element calculations. In this case, a singularity can be evidenced by observing the stress increase at a singular point when the grid mesh is refined. It is also possible to fit a function (2) characterised by a stress intensity $K$ and a singular order $n$ on 
the stress profiles. The singularity order is found to be very low: 0.01 and 0.04 on both sides of the interface, indicating a weak singularity.

It should be noted that $\mu$-XRD technique measures a mean value of the strain along the depth of the implanted layer. In order to compare measurements with simulations, the finite element results are averaged along the implanted layer thickness $d h$. Since the singularity order is low, this operation constitutes a mathematical regularisation of the stress field, and eliminates therefore the singularities. This is shown in figure 4 on two stress profiles taken along the $x$ direction: the stress values on the free surface in red, and the averaged values in blue.

Influential parameters. Many parameters influence the grains interaction: the implanted layer thickness $d h$, the direction of the grain boundary (angle $\alpha$ in Figure 3), and the grains orientations.

The implanted layer thickness $d h$ increases with the ion implantation energy, between 0.4 and $1.3 \mu \mathrm{m}$ for energies of respectively 60 and $500 \mathrm{keV}$. Two simulations are done for these two thicknesses, plus one for a much thinner layer $(0.05 \mu \mathrm{m})$. In all these cases the size in the $x$ direction is fixed at $d=15 \mu \mathrm{m}$. The crystal orientation of Grain 1 (figure 3) is [001], it is rotated by $20^{\circ}$ around $y$ axis for Grain 2 . The resulting strain profiles are presented in Figure 5. Here we show the component $\xi_{z z}$ of the displacement gradient which corresponds to the strain $\epsilon_{z z}$ normal to sample surface. For the smallest thickness (red curve), the displacement gradient values are close to the values predicted by the semi-analytical independent grain model (dashed lines). Near the boundaries, the grains interact, and the result differs from the semi-analytical model. For a $0.4 \mu \mathrm{m}$ layer thickness, the grains interaction propagates toward the grain centre. For higher thickness, $1.3 \mu \mathrm{m}$, the difference between the finite element results and the semi-analytical model (dashed lines) is higher. These simulations show that increasing the implantation energy, i.e. the implantation depth, increases the grains interaction near the grain boundaries and that the interaction propagates toward the grain centre. Another feature is noticeable: the mechanical interaction enlarges the strain difference in two neighbouring grains.

To avoid the combination of the grains interaction from both sides of each grain in the next simulations, the grain size $d$ is now set to $300 \mu \mathrm{m}$ for an implanted depth $d h$ of $1 \mu \mathrm{m}$, so that the displacement gradient in the grain centre reaches the values predicted by the independent grain model.

To study the effect of the grains orientation, the orientation of Grain 1 
in Figure 3 is fixed to [001], while the orientation of Grain 2 is rotated by an angle $\phi$ around the axis $y$. The grain boundary inclination is fixed to $\alpha=90^{\circ}$. The resulting strain profiles for three values of the rotation angle $\phi$ $\left(10^{\circ}, 20^{\circ}\right.$ and $\left.45^{\circ}\right)$ are shown in Figure 6 . The grains misorientation increases the displacement gradient gap between the two grains. This can create a maximum at a distance of 1 or $2 \mu \mathrm{m}$ from the grain boundary. The simulated strain maximum difference (blue curve) can reach twice the difference given by the independent grains model (dashed lines).

The misorientation is now fixed to $\phi=20^{\circ}$, and the grain boundary direction, i.e. the parameter $\alpha$ in Figure 3, can now vary between $70^{\circ}$ down to $10^{\circ}$. At the observed boundary, Grains 1 and 2 make respectively obtuse and acute angles with the free surface. The resulting strain profiles are presented in Figure 7. The effect of the boundary inclination only affects Grain 2 with the acute angle. The mechanical fields in Grain 2 are indeed sensitive to a quantity of Grain 1 material progressing under Grain 2 with the angle $\alpha$. The maximum strain in Grain 2 disappears for an angle $\alpha$ less than $30^{\circ}$.

These simulations confirm the observations of the strain field maps of Figure 2: for the considered implantation energies of 60 and $500 \mathrm{keV}$, i.e. for the corresponding strained layer thickness of 0.4 and $1.3 \mu \mathrm{m}$, the strain field is mainly determined by the grain orientation as predicted by the independent grains model. However, the mechanical interaction of the neighbouring grains affect the results. The interaction increases the strain differences between the grains, with a maximum located at distance of 1 or 2 times the implantation depth $d h$, and it propagates over a distance exceeding 50 times the implantation depth $d h$ toward the grain centre. For the considered implantation energies of 60 and $500 \mathrm{keV}$, with a grain size of about $18 \mu \mathrm{m}$, the interaction affects strain field even at the grain centre. Hence, in a polycrystal, the mechanical strain field of each grain results from its interaction with all the surrounding grains.

\section{Comparison to experimental results}

The strain field, measured by $\mu$-XRD on the two implanted samples, can now be compared with the finite element simulations. This comparison will focus on a profile, crossing two neighbouring grains, marked by a white arrow on the maps in Figure 2. The simulations will be done using the finite element model, described in Figure 3. The orientations of Grains 1 and 2 are 
determined from the Laue pattern. Before the comparison we must be aware of the following calculation simplifications, and their consequences:

- In the model, the swelling $s$ is supposed uniform, located in a subsurface layer of thickness $d h$. Previous studies [13, 14, 16], show that this hypothesis can hold for a $60 \mathrm{keV}$ implantation, but with less precision for a $500 \mathrm{keV}$ implantation. The mechanical loading imposed in the finite element simulations does not represent completely the real case.

- The geometry described by the model presented in Figure 3 is very simple and consists of a slice of two grains, periodically repeated. All the other surrounding grains are thus neglected. However, we know from the above simulations that the influence distance of a neighbouring grain is greater than the grain size.

- The whole geometry of the grains, and in particular their shape under the surface, is unknown. Hence, the boundary inclination between Grains 1 and 2 is to be estimated by varying the angle $\alpha$, and looking for the simulation estimating at best the experimental profile.

The first application is done on the polycrystal implanted at low energy (60 keV). We choose to simulate a profile crossing the two largest grains, indicated by the white arrow in Figure 2(a). The normal component $\xi_{z, z}$ and one of the shear components $\xi_{y, z}$ are shown in figure 8. Both experimental and simulated profiles behave similarly, but the match cannot be perfect because of the above remarks.

The second application is done on the $500 \mathrm{keV}$ implanted sample, with a larger implanted layer thickness, therefore with a stronger grain interaction. Again, the chosen profile, indicated by the white arrow in Figure 2(b), crosses the two largest grains. The corresponding curves for components $\xi_{z, z}$ and $\xi_{y, z}$ are presented in Figure 9. Despite the greater interaction, the simulation reproduces well the experimental results.

The finite element model, with its simplifications, can reproduce the main features of the mechanical interaction of neighbouring grains, observed on the experimental measurement. This encouraging result validates both the measurement methodology and the proposed mechanical model. 


\section{Conclusion}

Ion implantation is used to load mechanically a thin surface layer in a ceramic polycrystal. The resulting strain in the implanted layer is then measured by X-ray micro-diffraction in Laue mode with a spatial resolution of about 1 micrometer, enabling to map accurately the strain field inside a dozen of grains. The resulting displacement gradient has three main components $\xi_{x, z}, \xi_{y, z}$ and $\xi_{z, z}$, which depend mainly on the grain orientation and can be modelled by a semi-analytical mechanical model.

Mechanical grain interactions are observed in the 2D experimental strain maps near the grains boundaries, which can be reproduced adequately with a simplified finite element model. The simulations show that these interactions depend on the grain boundary inclination and on the relative grain orientation. They increase with the implantation depth, and propagate from the boundaries to the grain centre. Even at the centre of the grains, the actual strain differs from the prediction of the semi-analytical model.

A stress singularity appears at the intersection of the grain boundary with the free surface, but cannot be measured, because it disappears due to its low order and the averaging process along the depth probed by the $\mathrm{X}$-ray diffraction. In these areas, high stress levels can be reached, that can explain some phenomena occurring near the grain boundaries, for instance, accelerated release of helium [21].

The combination of ion implantation and X-ray micro-diffraction in Laue mode is thus a way to access at a microscopic scale the mechanical behaviour of the grains, and their mutual mechanical interactions. The results obtained in $\mathrm{UO}_{2}$ also apply for other ion implanted ceramic polycrystals if the following implantation conditions are verified: the implanted layer must be much smaller than the grain size, and negligible compared to the sample thickness; the mechanical strain model applies far from the sample edges.

\section{Acknowledgements}

We are grateful for the COSTO project managed by CEA and EDF for supporting this work.

\section{References}

[1] A. H. Agajanian, Ion implantation in microelectronics: a comprehensive bibliograph, New York : IFI/Plenum, 1981. 
[2] B. Radiguet, A. Barbu, P. Pareige, Understanding of copper precipitation under electron or ion irradiations in $\mathrm{FeCu} 0.1 \mathrm{wt} \%$ ferritic alloy by combination of experiments and modelling, Journal of Nuclear Materials 360 (2) (2010) 104-117.

[3] J. Pakarinen, M. Khafizov, L. He, C. Wetteland, J. Gan, A. T. Nelson, D. H. Hurley, A. El-Azab, T. R. Allen, Microstructure changes and thermal conductivity reduction in $\mathrm{UO}_{2}$ following $3.9 \mathrm{MeV} \mathrm{He}^{2+}$ ion irradiation, Journal of Nuclear Materials 454 (1-3) (2014) 283 - 289.

[4] P. Garcia, G. Martin, P. Desgardin, G. Carlot, T. Sauvage, C. Sabathier, E. Castelier, H. Khodja, M.-F. Barthe, A study of helium mobility in polycrystalline uranium dioxide, J. Nucl. Mater. 430 (2012) 156-165.

[5] A. Debelle, A. Boulle, F. Garrido, L. Thomé, Strain and stress buildup in He-implanted $\mathrm{UO}_{2}$ single crystals: an X-ray diffraction study, J. Mater. Sci. 46 (2011) 4683-4689.

[6] O. Ulrich, X. Biquard, P. Bleuet, O. Geaymond, P. Gergaud, J. S. Micha, O. Robach, F. Rieutord, A new white beam X-ray microdiffraction setup on the BM32 beamline at the European Synchrotron Radiation Facility, Rev. Sci. Instrum. 82 (2011) 033908.

[7] J.-S. Chung, G. Ice, Automated indexing for texture and strain measurement with broad-bandpass x-ray microbeams, J. Appl. Phys. 86 (1999) 5249 .

[8] A. MacDowell, R. Celestre, N. Tamura, R. Spolenak, B. Valek, W. Brown, J. Bravman, H. Padmore, B. Batterman, J. Patel, Submicron X-ray diffraction, Nuclear Instruments and Methods in Physics Research A 467-468 (2001) 936-943.

[9] F. Hofmann, D. Nguyen-Manh, M. Gilbert, C. Beck, J. Eliason, A. Maznev, W. Liu, D. Armstrong, K. Nelsond, S. Dudarev, Lattice swelling and modulus change in a helium-implanted tungsten alloy: $\mathrm{X}-$ ray micro-diffraction, surface acoustic wave measurements, and multiscale modelling, Acta Materialia 89 (2015) 352-363.

[10] A. Richard, H. Palancher, E. Castelier, J.-S. Micha, M. Gamaleri, G. Carlot, H. Rouquette, P. Goudeau, G. Martin, F. Rieutord, J. Piron, 
P. Garcia, Strains in light-ion-implanted polycrystals: influence of grain orientation, J. Appl. Cryst. 45 (2012) 826-833.

[11] M. Ibrahim, E. Castelier, H. Palancher, M. Bornert, S. Caré, J.-S. Micha, Laue pattern analysis for 2D strain mapping in light ion implanted polycrystals, Journal of Applied Crystallography 48 (2015) 990-999.

[12] T.-H. Nguyen, A. Debelle, A. Boulle, F. Garrido, L. Thom, V. Demange, Mechanical response of $\mathrm{UO}_{2}$ single crystals submitted to low-energy ion irradiation, J. Nucl. Mater. 467 (2015) 505-511.

[13] H. Palancher, P. Goudeau, A. Boulle, F. Rieutord, V. Favre-Nicolin, N. Blanc, G. Martin, J. Fouet, C. Onofri, Strain profiles in ion implanted ceramic polycrystals: An approach based on reciprocal-space crystal selection, Applied Physics Letters 108 (2016) 031903.

[14] H. Palancher, R. Kachnaoui, G. Martin, A. R. J.-C. Richaud, C. Onofri, R. Belin, A. B. H. Rouquette, C. Sabathier, G. Carlot, P. D. T. Sauvage, F. Rieutord, J. Raynal, P. Goudeau, A. Ambard, Strain relaxation in He implanted $\mathrm{UO}_{2}$ polycrystals under thermal treatment: An in situ XRD study, Journal of Nuclear Materials 476 (2016) 63-76.

[15] J. Ziegler, J. Biersack, M. Ziegler, SRIM the stopping and range of ions in matter, http://www.srim.org/ : http://www.srim.org, 1985.

[16] M. Ibrahim, Étude du comportement mécanique $\mathrm{dUO}_{2}$ implanté en hélium par micro-diffraction des rayons $\mathrm{X}$ et modélisation par éléments finis, Ph.D. thesis, Paris-Est University (2015).

[17] I. Fritz, Elastic Properties of $\mathrm{UO}_{2}$ at High Pressure, J. Appl. Phys. 47 (1976) 4353.

[18] V. Hein, F. Erdogan, Stress singularities in a two-material wedge, International Journal of Fracture Mechanics 7 (3) (1971) 317-330.

[19] D. Bogy, Two edge-bonded elastic wedges of different materials and wedge angles under surface tractions, Journal of Applied Mechanics 38 (2) (1971) 377-386.

[20] R. Zwiers, T. Ting, R. Spilker, On the logarithmic singularity of freeedge stress in laminated composite under uniform extension, Journal of Applied Mechanics 49 (1982) 561-569. 
[21] G. Martin, P. Garcia, C. Sabathier, G. Carlot, T. Sauvage, P. Desgardin, C. Raepsaet, H. Khodja, Helium release in uranium dioxide in relation to grain boundaries and free surfaces, Nucl. Instr. and Meth. B 268 (2010) 2133-2137.

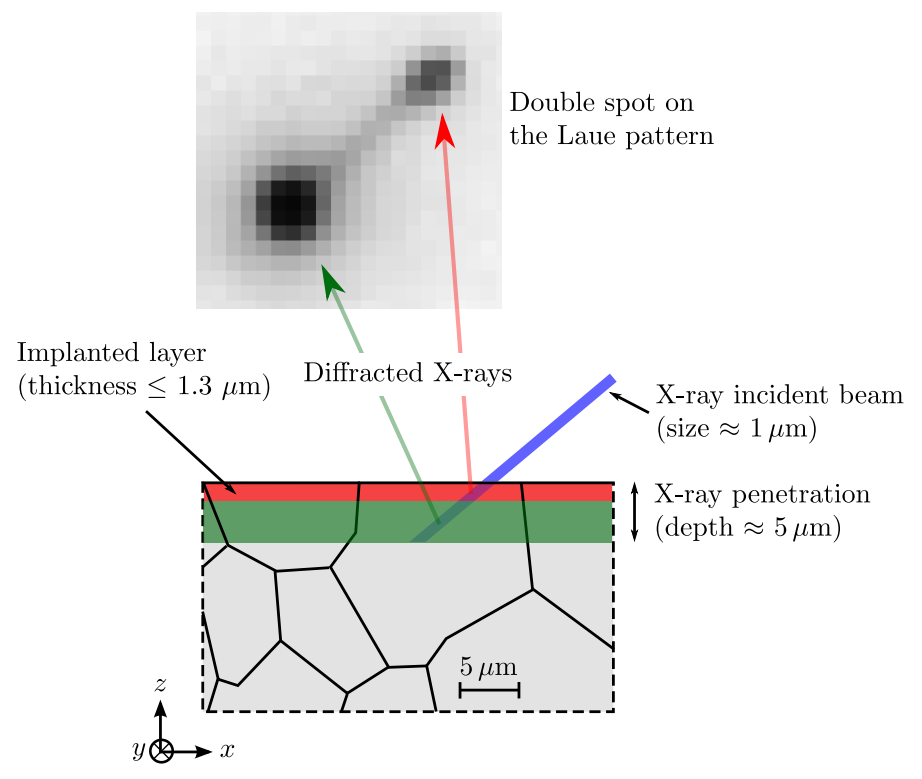

Figure 1: Schematic representation of a polycrystalline sample implanted in a thin surface layer, coloured in red. The X-ray penetration depth is coloured in green. The X-ray incident beam is diffracted by the implanted layer and the strain free substrate, producing double spots on the Laue pattern. 


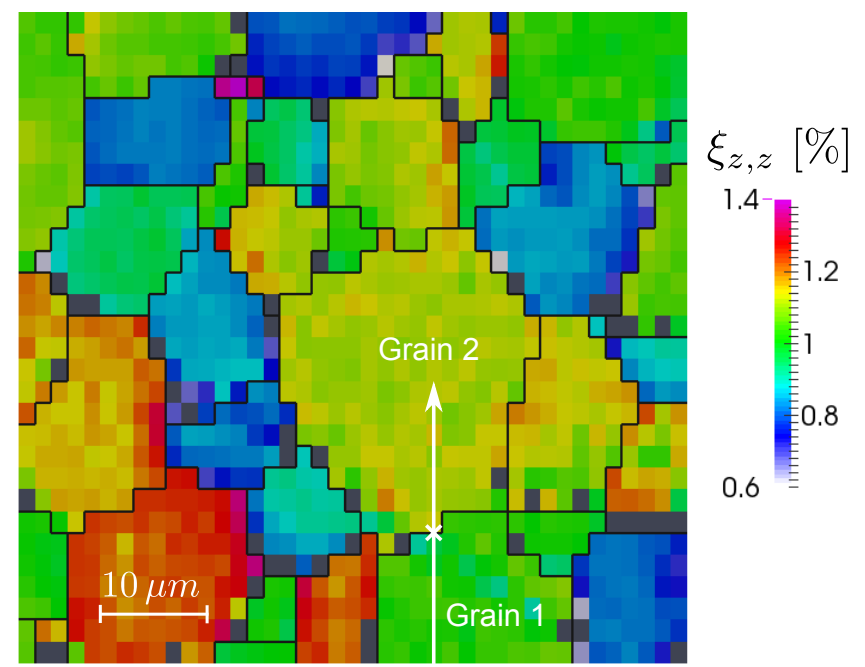

(a) $60 \mathrm{keV}-10^{16}$ ions $/ \mathrm{cm}^{2}$ He-implantation

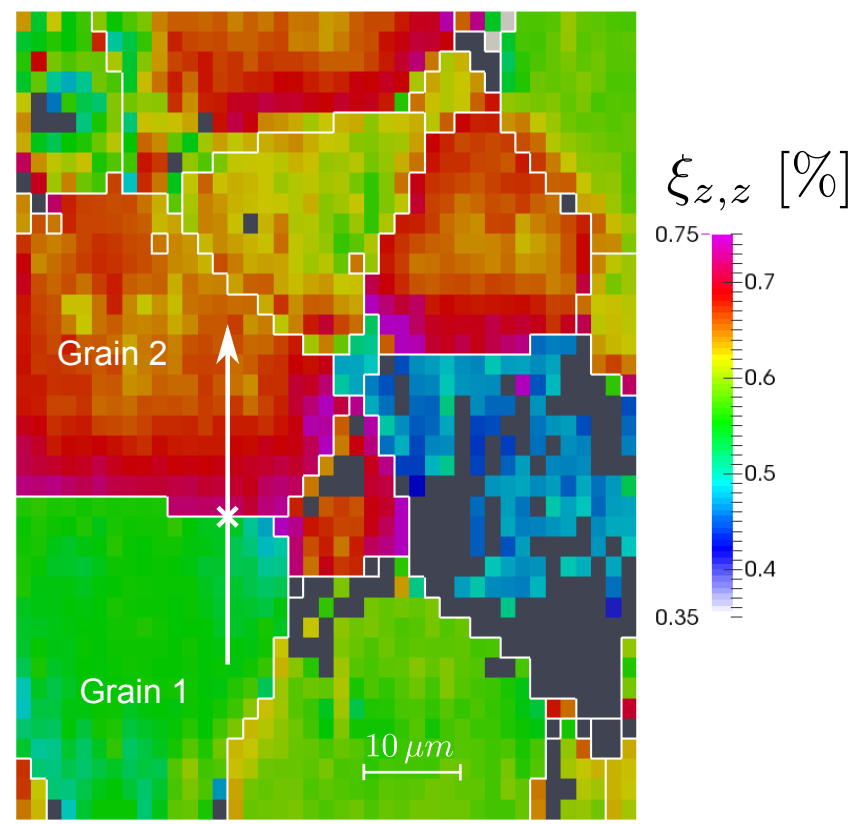

(b) $500 \mathrm{keV}-10^{16}$ ions $/ \mathrm{cm}^{2}$ He-implantation

Figure 2: Mapping of the displacement gradients normal component $\xi_{z, z}$ measured by $\mu$-XRD technique [11]. Two $\mathrm{UO}_{2}$ polycrystals are implanted with helium ions at $60 \mathrm{keV}$ - $10^{16}$ ions $/ \mathrm{cm}^{2}$ (a) and $500 \mathrm{keV}-10^{16}$ ions $/ \mathrm{cm}^{2}$ (b). 


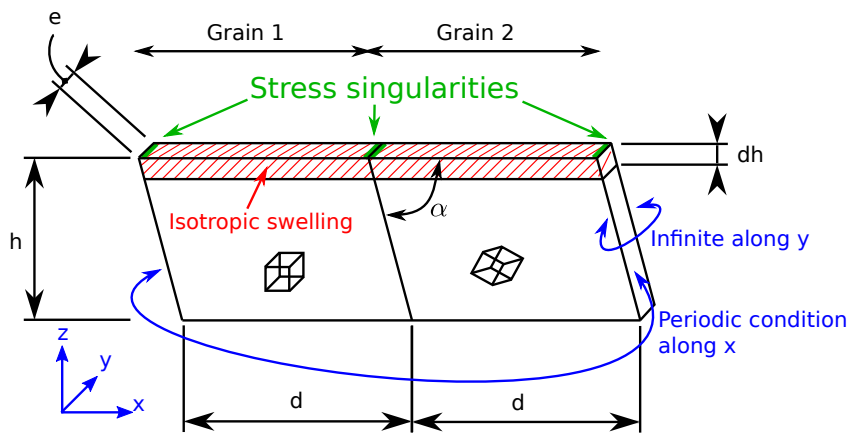

Figure 3: Geometry, boundary conditions, and loading condition used in a simulation of two grains implanted in a thin surface layer.

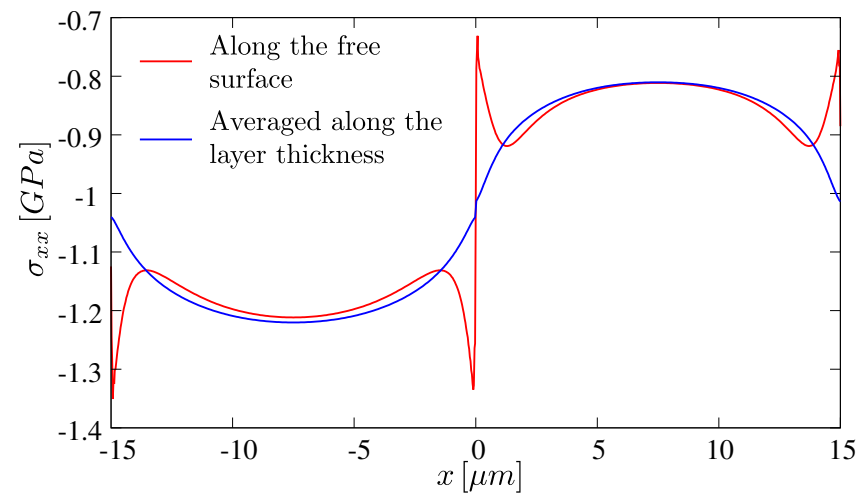

Figure 4: Profiles of the simulated stress component $\sigma_{x x}$ along the free surface (red curve), and its mean value along the strained layer thickness (blue curve). 


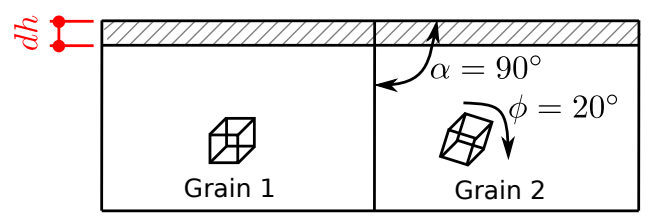

(a)

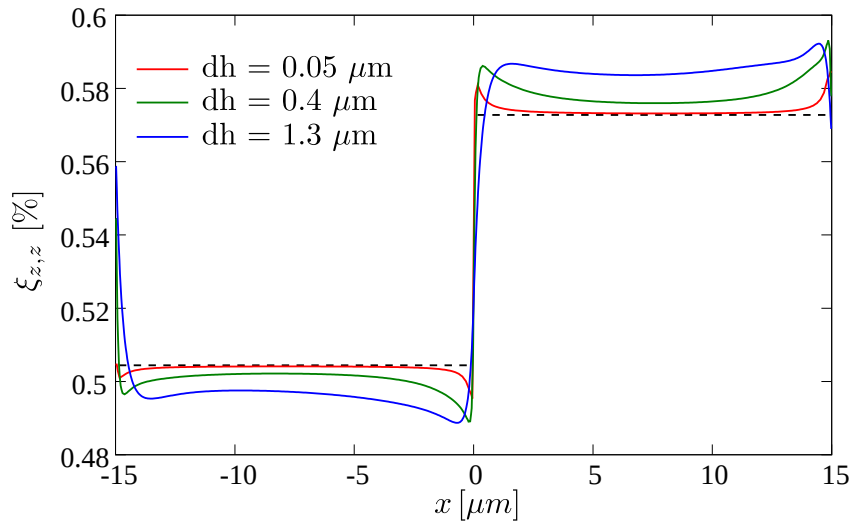

(b)

Figure 5: (a) Schematic representation, (b) and simulated displacement gradient component $\xi_{z, z}$ profiles (solid lines) for three implantation depths $d h$. The dashed lines represent the semi-analytical grain independent model. 


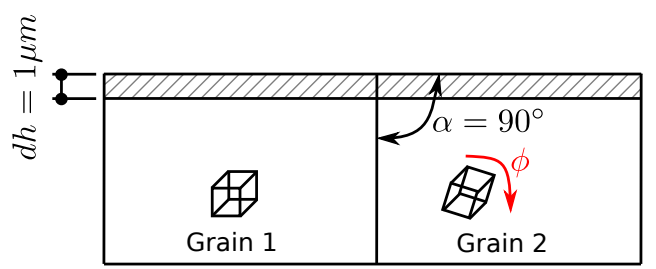

(a)

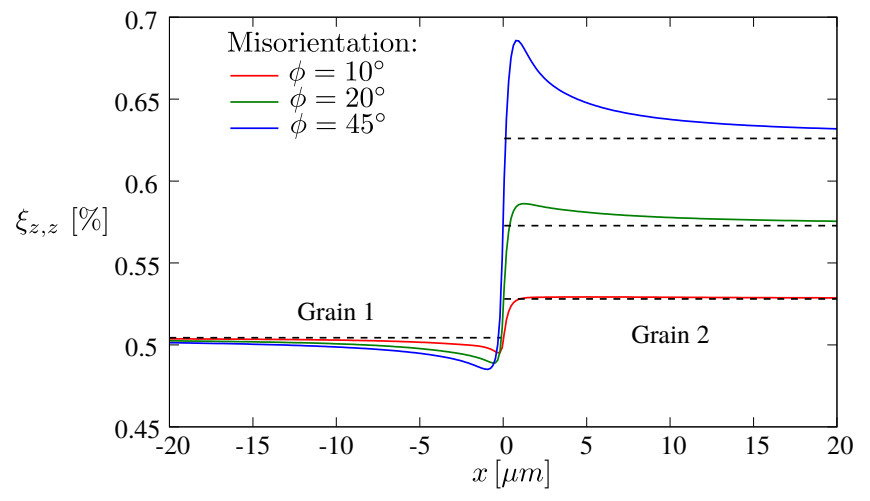

(b)

Figure 6: (a) Schematic representation, (b) and simulated displacement gradient component $\xi_{z, z}$ profiles (solid lines) for three different misorientations $\phi$ of Grain 2. The dashed lines represent the semi-analytical grain independent model. 


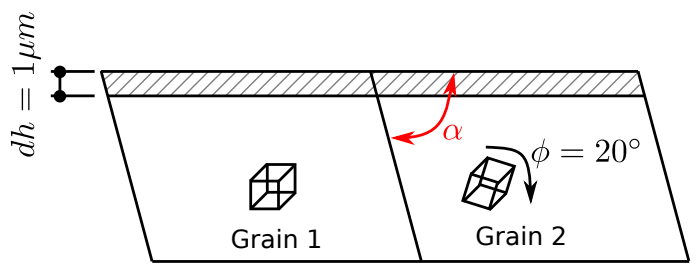

(a)

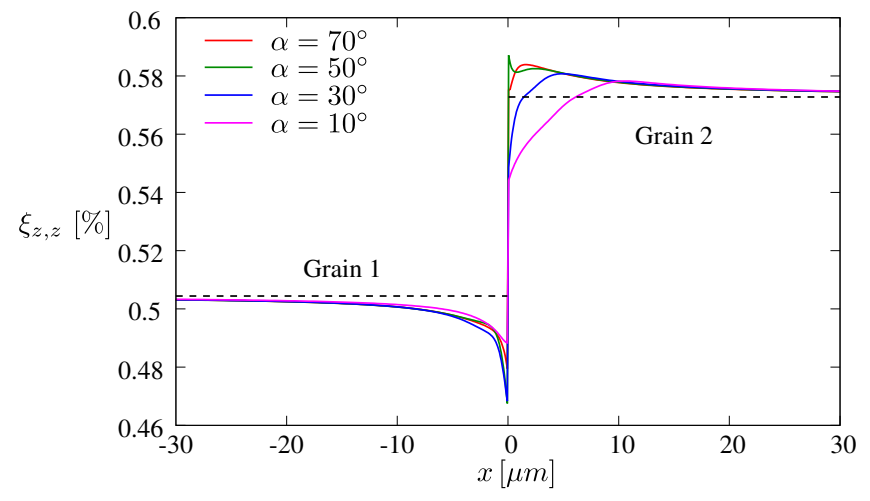

(b)

Figure 7: (a) Schematic representation, (b) and simulated displacement gradient component $\xi_{z, z}$ profiles (solid lines) for four different grain boundary orientations $\alpha$. The dashed lines represent the semi-analytical grain independent model. 


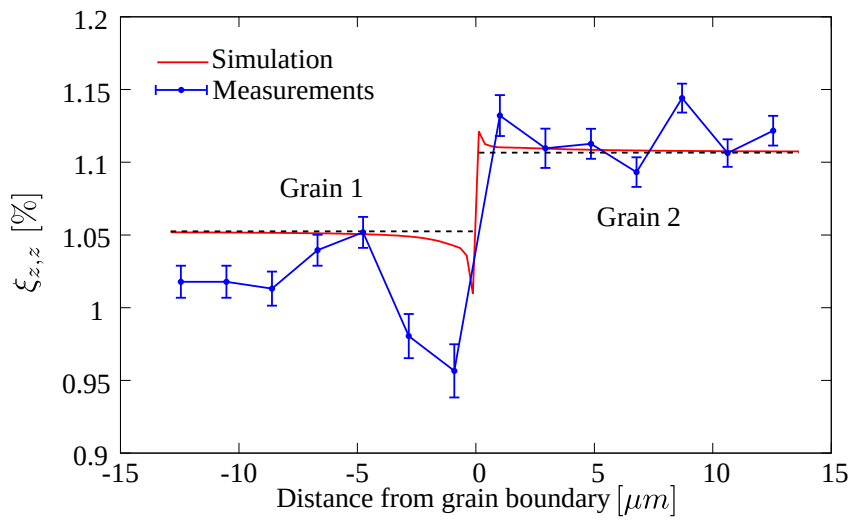

(a)

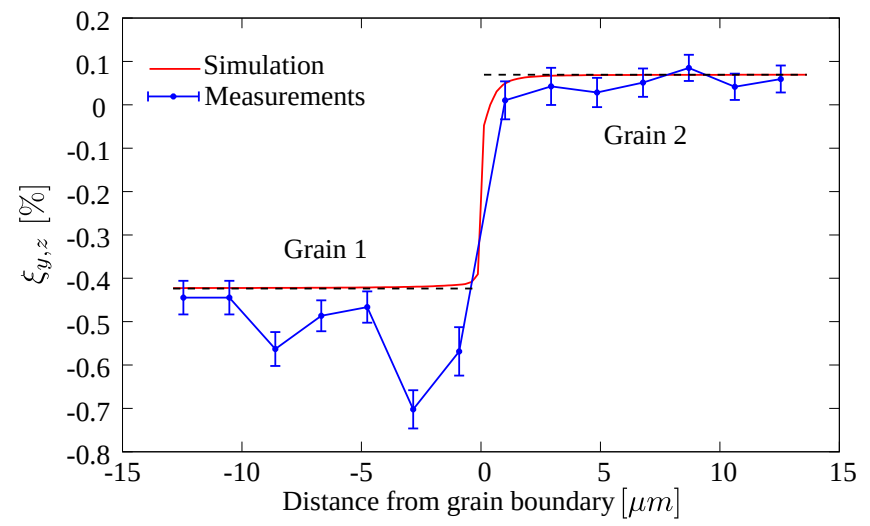

(b)

Figure 8: Profiles of components $\xi_{z, z}$ and $\xi_{y, z}$ taken in the $60 \mathrm{keV}$ implanted sample along the white arrow of figure 2(a). Comparison between $\mu$-XRD measured values (blue) and finite element simulations (red). The dashed lines represent the semi-analytical model. 


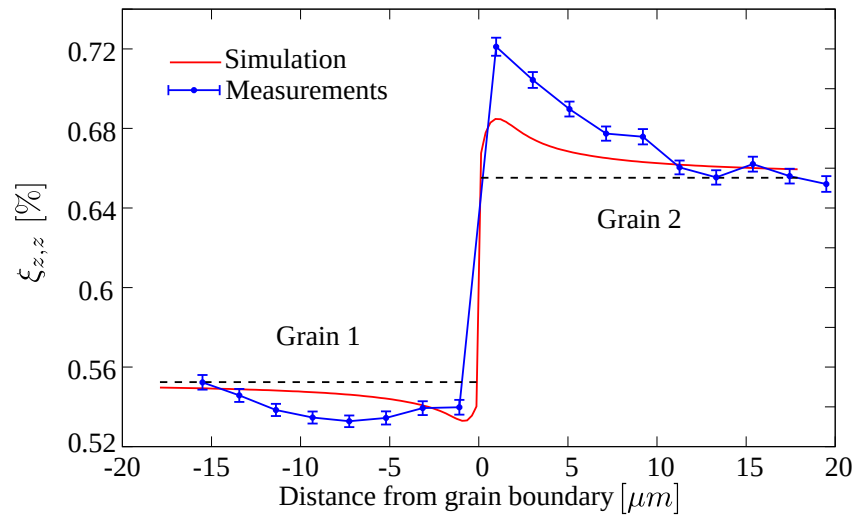

(a)

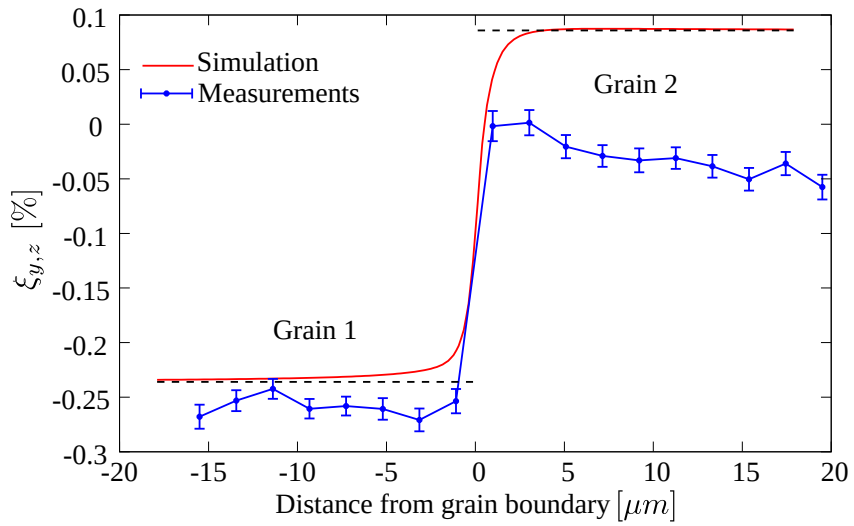

(b)

Figure 9: Profiles of components $\xi_{z, z}$ and $\xi_{y, z}$ taken in the $500 \mathrm{keV}$ implanted sample along the white arrow of figure 2(b). Comparison between $\mu$-XRD measured values (blue) and finite element simulations (red). The dashed lines represent the semi-analytical model. 